\title{
Radiation Dose Reduction in 4D Cerebral CT Angiography by Individualized Estimation of Cerebral Circulation Time
}

\author{
(D) M.R. Radon, (D)A. Chandran, (D) M. Bhojak, and (D).V. Das
}

\begin{abstract}
BACKGROUND AND PURPOSE: The novel technique of 4D CTA for dynamic assessment of the intracranial vessels has a greater radiation burden than conventional CTA. Previous descriptions of the technique used a fixed-duration exposure protocol. This study examines the potential for dose reduction by individualizing exposure time to patient physiology by the use of time-enhancement curve techniques as previously applied in CT angiography and venography.
\end{abstract}

MATERIALS AND METHODS: 4D-CTA examinations performed at our institution were retrospectively reviewed. Scan protocols used a test-bolus scan with either a subjective estimate of the main acquisition timing (estimated-duration method) or a quantitative measure (measured-duration method). The estimated-duration method used peak arterial enhancement to determine the start of exposure, with the duration chosen at the radiologist's discretion. The measured-duration method used arterial and venous time-enhancement curves to determine exposure start and duration. Exposure duration, study adequacy, quality score, and maximum venous enhancement were compared among groups.

RESULTS: One hundred fifty-one examinations used the estimated-duration method, and 53 used measured-duration. The measuredduration method used a shorter exposure time (10 versus 15.8 seconds; $P<.001$ ). There was no statistically significant difference in the study adequacy rate, subjective quality score, or maximum venous enhancement. The radiation dose was reduced by $51 \%$ in the measuredduration method $(3021 \mathrm{mGy} \times \mathrm{cm}, 6.9 \mathrm{mSv}$, versus $1473 \mathrm{mGy} \times \mathrm{cm}, 3.4 \mathrm{mSv})$. Both methods showed good agreement with DSA $(\kappa=0.88$ for estimated-duration, $\kappa=1.0$ for measured duration).

CONCLUSIONS: Exposure time in 4D-CTA can be reduced with dual time-enhancement curves to match exposure to physiology without degrading study adequacy or quality.

ABBREVIATIONS: $\mathrm{t}_{\mathrm{OA}}=$ time to onset of arterial enhancement; $\mathrm{t}_{\mathrm{PA}}=$ time delay to peak arterial enhancement; $\mathrm{t}_{\mathrm{PV}}=$ time to peak venous enhancement

$\mathbf{T}$ he recent development of time-resolved 4D-CTA provides a new technique for the assessment of intracranial flow dynamics, which can serve as a less invasive alternative to intra-arterial DSA. Potential uses for this technique include assessment of dural arteriovenous fistulas $^{1-4}$ and arteriovenous malformations ${ }^{5}$ and classification of vessel clot burden and collateral flow in occlusive stroke. $^{6,7}$

Due to the multiphasic nature of $4 \mathrm{D}-\mathrm{CTA}$, the radiation burden is higher than in a single-phase study. Concern over the total dose has previously been raised for CT perfusion, ${ }^{8,9}$ and due to the

Received March 10, 2016; accepted after revision July 5.

From the Department of Neuroradiology, The Walton Centre, Liverpool, United Kingdom.

Please address correspondence to Mark Radon, MD, Department of Neuroradiology, The Walton Centre, Liverpool, L9 7UJ, UK; e-mail: Mark.radon@thewaltoncentre. nhs.uk

http://dx.doi.org/10.3174/ajnr.A4911 similarity of the techniques, it carries over to 4D-CTA. The effective dose for 4D-CTA reported in the literature ranges between 5.1 and $5.62 \mathrm{mSv} .^{2,3,5,10}$ These previously described techniques use a dynamic acquisition with a predetermined exposure time (between 15 and 22 seconds), with or without a preceding test-bolus acquisition. In addition to variance in exposure time, there is also variability in the rotation period and, therefore, temporal resolution. Earlier literature reports used a 1000-ms rotation period, but more recent studies have cited shorter rotation periods of 500 and $350 \mathrm{~ms}$, though using a higher tube current. ${ }^{3,10,11}$

Test-bolus techniques yielding a time-attenuation curve have previously been shown beneficial in both $3 \mathrm{D}-\mathrm{CTA}^{12}$ and CT venography, ${ }^{13}$ by allowing the time of acquisition to be matched to the time of peak contrast enhancement within the vessels of interest. A similar approach has been applied in previous descriptions of 4D-CTA to trigger the start of the acquisition. The benefit of accurate timing in 4D-CTA is not on peak enhancement, but 
instead, it assists in achieving adequate temporal coverage. While an arterial time-enhancement curve provides information about contrast arrival, the venous time-enhancement curve provides information about contrast washout. We hypothesized that knowledge of these parameters can be used to tune the acquisition duration and provide an opportunity for dose reduction, while simultaneously avoiding inadequate studies in patients with a slow cerebral circulation time.

The primary end point of this study was to demonstrate diagnostic equivalence and reduced exposure time by using the measured-duration method in comparison with the estimated-duration method.

\section{MATERIALS AND METHODS \\ Study Design}

4D-CTA has been in use locally in selected patients since November 2013. Following a routine audit of radiation doses, which showed doses higher than published reports from other institutions, the CT protocol was modified in January 2015. The exposure factors were shown to be higher than those in prior literature reports, and previously published parameters were adopted as part of the review. ${ }^{1}$

At the same time, reducing exposure durations was attempted. The scan protocol was changed from using a subjective assessment of a test-bolus acquisition (estimated-duration method) to a replacement protocol using quantitative, objective measurements from the test bolus (measured-duration method). Patients were identified by searching the radiology information system for 4D-CTA examinations performed from November 2013 to July 2015. Retrospective analysis of these studies was performed to determine the adequacy of the method.

Because this study represents a retrospective review of these methods, the institutional research and development department waived the requirement for regional ethics committee approval or informed consent.

\section{CT Scanner and Scan Parameters}

All studies were performed by using a 320-section CT scanner with maximum 160-mm volume coverage capability. A 3-phase protocol was used, consisting of a test-bolus, unenhanced subtraction mask, and dynamic acquisition. The test bolus used 15 $\mathrm{mL}$ of iodinated contrast (iopamidol, Niopam 370; Bracco UK, High Wycombe, UK) given intravenously at $6 \mathrm{~mL} / \mathrm{s}$, followed by $30 \mathrm{~mL}$ of saline at $6 \mathrm{~mL} / \mathrm{s}$. Repeat single 2 - $\mathrm{mm}$ sections $(80 \mathrm{kV}, 100$ $\mathrm{mAs}$ ) through the circle of Willis at 2-second intervals were performed from which time-enhancement curves were derived. The mask acquisition used a single volumetric acquisition of the whole head $(80 \mathrm{kV}, 300 \mathrm{mAs})$.

Scan parameters for the estimated-duration protocol were those determined during the initial protocol development $(80 \mathrm{kV}$, $150 \mathrm{~mA}, 750 \mathrm{~ms}$ ) and were determined on the basis of local preference and empiric assessment of adequacy. Parameters for the measured-exposure protocol were based on a prior literature report $(80 \mathrm{kV}, 100 \mathrm{~mA}, 1000 \mathrm{~ms}) .{ }^{1}$ In both protocols, the temporal reconstruction interval was $500 \mathrm{~ms}$. The $\mathrm{z}$-axis coverage was the whole head from the vertex to $\mathrm{C} 1$ and was usually the maximum,
$160 \mathrm{~mm}$. A total of $35 \mathrm{~mL}$ of contrast was given at $6 \mathrm{~mL} / \mathrm{s}$ for the main acquisition, followed by $30 \mathrm{~mL}$ of saline at $6 \mathrm{~mL} / \mathrm{s}$.

\section{Acquisition Timing}

An ROI was placed on a major artery (basilar or ICA) on the test-bolus images. The time delay between the start of contrast injection and the peak of arterial enhancement $\left(t_{P A}\right)$ was measured.

Estimated-Duration Protocol. The volume acquisition was triggered at a $t_{\mathrm{PA}}$ of 4 seconds. The exposure duration was at the discretion of the supervising radiologist, from a starting point of 16 seconds. This could be adjusted depending on clinical features and review of the arterial enhancement curve.

Measured-Duration Exposure Protocol. In addition to a major artery ROI, a second venous sinus ROI was used. The time delay to peak venous enhancement $\left(t_{\mathrm{PV}}\right)$ was measured, and the arterial ROI was used to determine the time to onset of arterial enhancement $\left(t_{\mathrm{OA}}\right)$, defined as an increase of attenuation of $100 \mathrm{HU}$ over baseline. Acquisition was triggered at the $\mathrm{t}_{\mathrm{OA}}$, and duration was set at $t_{\mathrm{PV}}$ to $\mathrm{t}_{\mathrm{OA}}$. This process is illustrated schematically, together with typical subtracted image appearances, in Fig 1.

\section{Image Analysis}

Study Adequacy. The dynamic acquisitions were examined for the visible inflow of contrast into the terminal ICA. Volumes at the start of the dynamic acquisitions that showed $<40 \mathrm{HU}$ of enhancement of the ICA were regarded as noncontributory. Volumes at the end of the acquisition that showed venous enhancement less than peak were also regarded as noncontributory. When the final volume showed rising venous enhancement, an adequate venous enhancement was defined arbitrarily as enhancement of $>200 \mathrm{HU}$. A study was also judged as having inadequate time coverage if no prepeak arterial volumes were acquired. A subjective diagnostic quality score was also recorded by 2 experienced neuroradiologists in consensus. A score of 1 indicated a nondiagnostic study (for any reason), and 5 indicated excellent quality.

Dose Reporting. Dose-length products for the mask, test bolus, and dynamic components of the study were recorded. Effective doses were computed by using the conversion factor of 2.3 $\mu \mathrm{Sv} / \mathrm{mGy} / \mathrm{cm}^{14}$

Concordance with DSA. When patients subsequently underwent DSA, this was used as the criterion standard for comparison. To minimize bias, we used the initial blinded radiology report for the $4 \mathrm{D}-\mathrm{CTA}$ as the basis of concordance. When there was discordance, the studies were reviewed to determine the cause. In view of the variety of pathology and indications for scanning, sensitivity and specificity were not calculated. Studies were considered concordant when both the DSA and 4D-CTA had the same category of diagnosis (eg, no vascular abnormality, AVF, AVM, aneurysm, vascular occlusion/stenosis).

\section{Statistical Analysis}

The estimated-duration and measured-duration methods were compared on parameters of exposure duration, peak venous enhancement, study adequacy rate, and subjective quality score. 

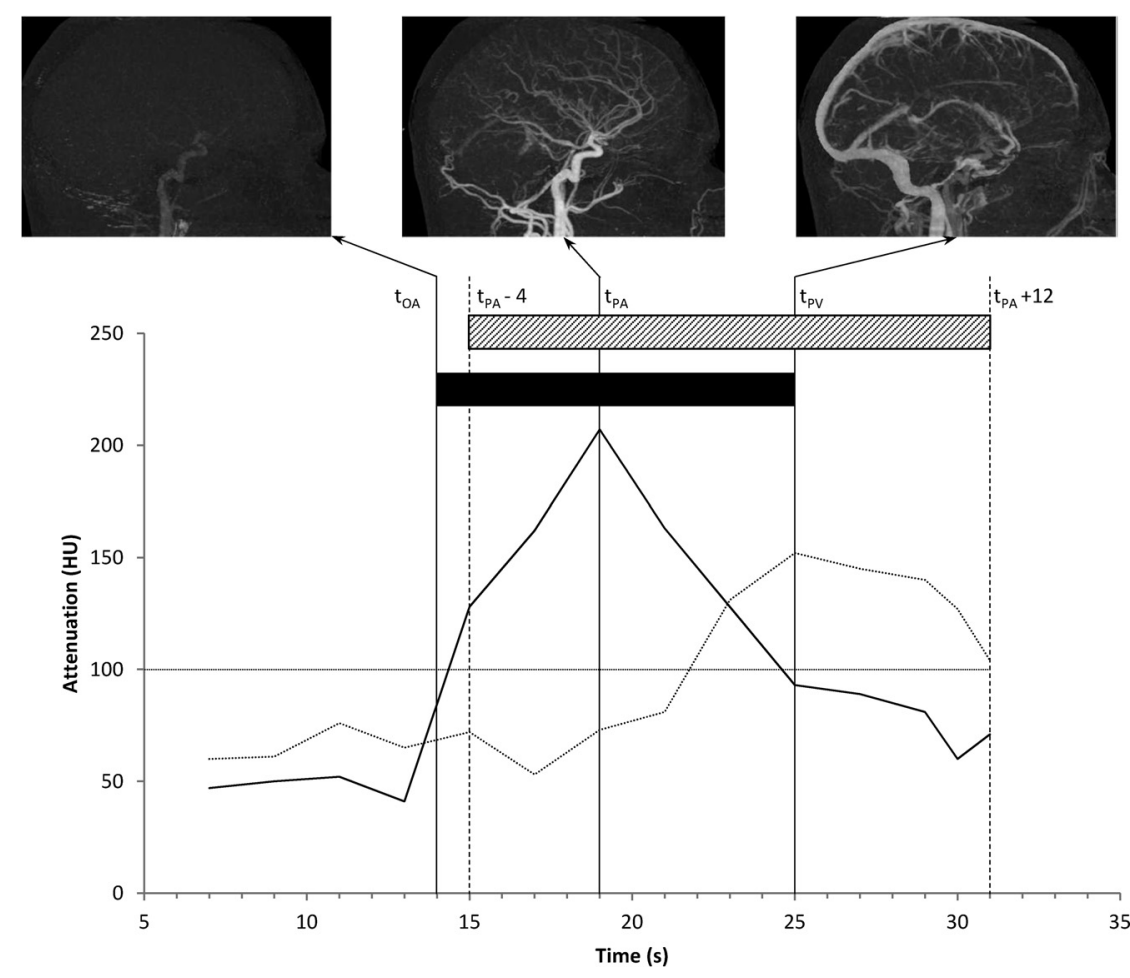

FIG 1. Schematic representation showing typical time-attenuation curves for arterial (solid line) and venous (dotted line) profiles. The $t_{\mathrm{OA}}$ was set to the nearest integer second in which the arterial attenuation crossed $100 \mathrm{HU}(14$ seconds in this figure, but note that measurements were only made on odd-numbered seconds). The $t_{P A}$ and $t_{P V}$ represent times of peak arterial and venous attenuation, respectively. The gray bar indicates the exposure time for the estimated duration protocol (16 seconds), and the black bar indicates the exposure time for the measuredduration protocol. Subtracted MIP images illustrate typical appearances of the acquisition at key phases.

Table 1: Demographics and breakdown of indications for imaging

\begin{tabular}{lcc}
\hline & $\begin{array}{c}\text { Estimated } \\
\text { Duration } \\
(\boldsymbol{n}=151)\end{array}$ & $\begin{array}{c}\text { Measured } \\
\text { Duration } \\
(\boldsymbol{n}=53)\end{array}$ \\
\hline $\begin{array}{l}\text { Demographics } \\
\text { Age (yr) }\end{array}$ & $53.4(19-87)$ & $51.8(21-79)$ \\
$\quad$ Sex (M/F) & $67: 84$ & $25: 28$ \\
Indications (No.) (\%) & & \\
$\quad$ Intraparenchymal hemorrhage & $47(31 \%)$ & $20(38 \%)$ \\
Suspected vascular lesion & $37(25 \%)$ & $11(21 \%)$ \\
Tinnitus & $21(14 \%)$ & $5(9 \%)$ \\
Tumor vascular assessment & $11(7 \%)$ & $5(9 \%)$ \\
Subdural hemorrhage & $5(3 \%)$ & $0(0 \%)$ \\
Subarachnoid hemorrhage & $4(3 \%)$ & $0(0 \%)$ \\
Untreated AVM/F assessment & $7(5 \%)$ & $5(9 \%)$ \\
$\quad$ Treated AVM/F assessment & $6(4 \%)$ & $3(6 \%)$ \\
$\quad$ Venous stenosis/thrombosis & $8(5 \%)$ & $3(6 \%)$ \\
$\quad$ (including assessment of idiopathic & & \\
$\quad$ intracranial hypertension) & & \\
Other & $5(3 \%)$ & $1(2 \%)$ \\
\hline
\end{tabular}

Note:-AVM/F indicates arteriovenous malformation or fistula.

Peak venous enhancement was compared by using the independent-samples $t$ test after confirming normality with the ShapiroWilk test. The Mann-Whitney $U$ test was used for non-normally distributed variables (exposure duration and study quality score), and the $\chi^{2}$ test, for study adequacy rates. The Bonferroni correction for multiple comparisons was applied to the above 4 tests, by multiplying $P$ values by the number of tests, subject to a maximum of 1.0. Statistical significance was defined as 5\% after cor- and indications for 4D-CTA are summarized in Table 1.

With the subjective method, 145 scans were performed by using 160-mm coverage, 5 were performed with 140-mm coverage, and 1 , with 150 -mm coverage. All scans performed with the objective method had 160 -mm coverage.

The measured-duration method used a shorter exposure time (10 versus 15.8 seconds, $P<.001$ ), acquired fewer volumes, and achieved lower radiation doses $(3021 \mathrm{mGy} \times \mathrm{cm}, 6.9 \mathrm{mSv}$, versus $1473 \mathrm{mGy} \times \mathrm{cm}, 3.4 \mathrm{mSv}$ ). These findings are summarized in Table 2. There was no statistically significant difference after correction for multiple comparisons in maximum venous enhancement, subjective study quality, or adequate time coverage rate. The distribution of maximum venous enhancement is illustrated in Fig 2. Both methods showed good intermodality concordance between $4 \mathrm{D}$-CTA and DSA $(\kappa=0.88$ for estimated duration, $\kappa=$ 1.0 for measured duration).

For examinations using the full $160-\mathrm{mm}$ anatomic coverage, the contributions to the radiation dose of the individual components of the examinations were the following: test bolus (doselength product $=84 \mathrm{mGy} \times \mathrm{cm}$, effective dose $=0.19 \mathrm{mSv}$ ), mask acquisition $(339 \mathrm{mGy} \times \mathrm{cm}, 25.2 \mathrm{mGy}, 0.78 \mathrm{mSv}$, CT dose in$\mathrm{dex}=25.2 \mathrm{mGy})$, collimated estimated-duration volume acquisition $(162 \mathrm{mGy} \times \mathrm{cm} / \mathrm{s}, 0.37 \mathrm{mSv} / \mathrm{s}, 10.1 \mathrm{mGy} / \mathrm{s})$, and measuredduration volume acquisition $(108 \mathrm{mGy} \times \mathrm{cm} / \mathrm{s}, 0.25 \mathrm{mSv} / \mathrm{s}, 6.8$ $\mathrm{mGy} / \mathrm{s})$. For studies with less coverage, doses for mask and main acquisitions were proportionally lower. 
Table 2: Diagnostic performance of original and measured-duration protocols ${ }^{\mathrm{a}}$

\begin{tabular}{|c|c|c|c|c|}
\hline & $\begin{array}{c}\text { Estimated } \\
\text { Duration } \\
(n=151)\end{array}$ & $\begin{array}{c}\text { Measured } \\
\text { Duration } \\
(n=53)\end{array}$ & $\begin{array}{c}P \\
\text { Value }\end{array}$ & $\begin{array}{l}\text { Adjusted } \\
P \text { Value }\end{array}$ \\
\hline \multicolumn{5}{|l|}{ Exposure and dose } \\
\hline Exposure time (sec) & $15.75(8.25-25.5)$ & $10.0(8-12)$ & $<.001$ & $<.001^{\mathrm{b}}$ \\
\hline No. of volumes & $31(26-36)$ & 19 (19-19) & & \\
\hline Dose-length product $(\mathrm{mGy} \times \mathrm{cm})$ & $3021(2536-3502)$ & $1473(1459-1526)$ & & \\
\hline Volume CT dose index (mGy) & $185(162-223)$ & $88.9(88.9-88.9)$ & & \\
\hline Effective dose (mSv) & $6.9(5.8-8.0)$ & $3.4(3.4-3.5)$ & & \\
\hline \multicolumn{5}{|l|}{ Study quality } \\
\hline Maximum venous enhancement (HU) & $372(352-392)$ & $324(294-353)$ & .019 & .076 \\
\hline Adequate time coverage (No.) & $134(88.7 \%)$ & $49(92.5 \%)$ & .444 & 1.00 \\
\hline Quality score & $5(5-5)$ & $5(5-5)$ & .837 & 1.00 \\
\hline No. of prearterial volumes & $6(4-10)$ & $0(0-1)$ & $<.001^{\mathrm{C}}$ & $<.001^{\mathrm{b}, \mathrm{c}}$ \\
\hline No. of postpeak venous volumes & $2(0-6)$ & $0(0-2)$ & $<.001^{\mathrm{C}}$ & $<.001^{\mathrm{b}, \mathrm{c}}$ \\
\hline \multicolumn{5}{|l|}{ Diagnostic performance } \\
\hline Positive findings (No.) & $58(38.4 \%)$ & $21(39.6 \%)$ & & \\
\hline No. having DSA & $56(37.1 \%)$ & $22(41.5 \%)$ & & \\
\hline $\begin{array}{l}\text { Intermodality concordance between } \\
\text { 4D-CTA and DSA (No.) }\end{array}$ & $51(91.0 \%)$ & $22(100.0 \%)$ & & \\
\hline Cohen $\kappa$ statistic & $\kappa=0.88$ & $\kappa=1.0$ & & \\
\hline
\end{tabular}

${ }^{a}$ Noncount values are shown as median (interquartile range), except for exposure time shown as median (range) and enhancement shown as mean $(95 \% \mathrm{Cl})$. Values for dose-length product include the entire examination, including test-bolus acquisition. CT dose index values include only whole-head acquisitions.

b Significant at the $5 \%$ level after Bonferroni correction.

c Post hoc test.

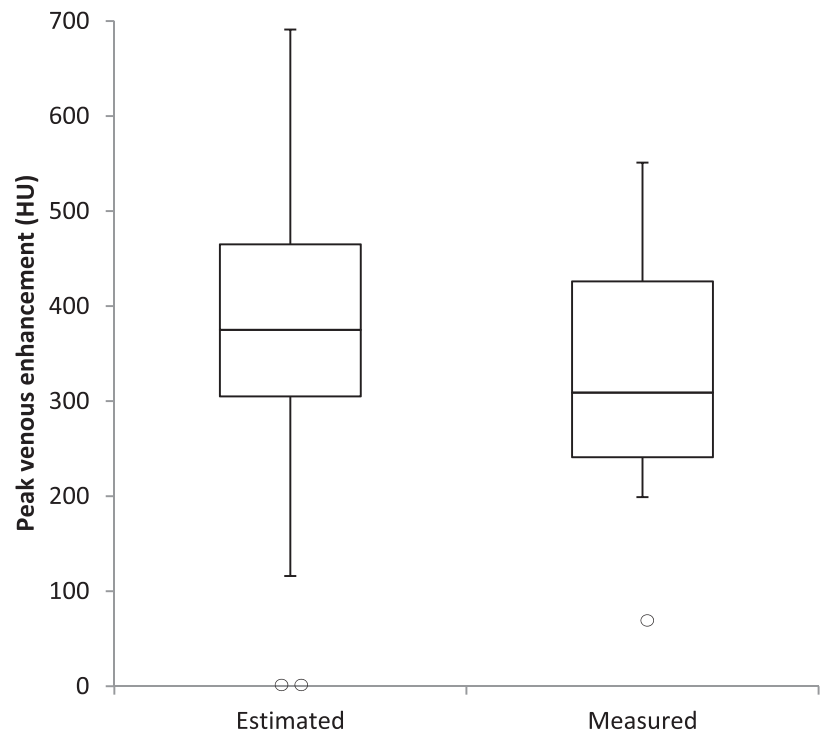

FIG 2. Boxplot illustrating distribution of peak venous enhancement between the estimated- and measured-duration scan protocols. Two outliers are shown in the estimated-duration group, in which no venous enhancement was present due to inadequate time coverage, and 1 outlier with very poor venous enhancement is shown in the measured-duration group.

\section{DISCUSSION}

The main aim of this study was to demonstrate a technique for reducing the radiation dose by reducing total exposure time, with the intention of discarding only data of minimal diagnostic value. Other approaches to controlling dose in CTA and CTP have been previously reported. These include the use of iterative reconstruction algorithms, which can reduce the dose while retaining image quality, ${ }^{15}$ and the use of a reduced tube potential $(80 \mathrm{kV})$ to improve contrast with better matching to the iodine K-edge. ${ }^{16}$ All prior reports of $4 \mathrm{D}-\mathrm{CTA}$ make use of both techniques.
The use of the measured-duration method presented here has shown a reduced exposure time both in comparison with our historical control data and prior literature (Table 3 ). In addition, our measured-duration method both reduced the tube current and increased the rotation time (and therefore reduced the temporal resolution) over and above the estimated-duration method. The overall effect on per-image exposure was small (112 versus $100 \mathrm{mAs}$ ) with no subjectively perceptible change in noise. Subjective assessment of study quality included the adequacy of temporal resolution for the detection of abnormalities. This was not thought to be different among techniques, possibly due to the retention of the same reconstruction interval of $500 \mathrm{~ms}$. A further small contribution to dose-saving was setting the rotation time to a multiple of the temporal reconstruction interval; when the total exposure time is not a multiple of the reconstruction interval, part of the final rotation may not be reconstructable. In the estimated-duration group, the contribution of this effect was $<1 \%$ of the total dose.

The recent description of higher temporal resolution techniques ${ }^{3,11,10}$ may enable better characterization of flow dynamics. However, these techniques are associated with increased noise, which can be offset by the use of a higher tube current. These higher dose techniques for 4D-CTA may particularly benefit from strict control of exposure duration.

In this series, the measured-exposure group showed a trend toward a lower mean maximum venous enhancement value. This can be attributed to acquisition terminating before peak venous enhancement. One potential limitation of defining the end of the acquisition as the $t_{\mathrm{PV}}$ on the test bolus is that the duration of the main injection ( 5 seconds) is longer than that of the test injection (2.5 seconds); therefore, peak venous enhancement may be later. Thus, the timing derived in the measured-duration method is only an estimate because timing is inferred from the test-bolus scan. This is illustrated in Fig 3, which shows that the distribution of the number of postvenous volumes is heavily skewed toward zero in both groups, indicating a tendency toward early termination. We propose that to further reduce the rate of inadequate studies, the end of the acquisition should be set at $t_{\mathrm{PV}}+1$ or $\mathrm{t}_{\mathrm{PV}}+$ 2 seconds. Even with this additional exposure time, the exposure duration would still be expected to be lower than that in the fixedduration methods previously reported (the mean effective dose reported in this study would be $3.65 \mathrm{mSv}_{\text {if }} \mathrm{t}_{\mathrm{PV}}+1$ had been used as the end of acquisition time). This additional late venous phase exposure could potentially be performed by using intermittent rather than continuous scanning. ${ }^{3}$

Figure $3 A$ shows the distribution of exposure duration within the 2 groups. The measured-duration group shows relatively tight clustering of duration around 10 seconds. The outlying study 
used 15 seconds, but this was due to incorrect measurement of the $t_{\mathrm{PV}}$. If this had been measured correctly, the duration would have been 12 seconds. The estimated-duration group did demonstrate 7 studies that used exposures of $>20$ seconds. In 6 of these cases, there were substantial intracranial hematomas; hence, a prolonged exposure was prescribed. However, in these cases, there were at least 10 postpeak venous volumes acquired, which indicated that the exposure could have been terminated up to 5 seconds earlier. In the final case, the patient had a history of cardiac failure and a severely prolonged circulation time so that venous enhancement was still rising at 25 seconds. There were no patients with similar features in the measured-duration group, which is a potential confounding factor.

In the measured-duration group, 4 studies showed inadequate time coverage. Three were due to inadequate venousphase images, and 1 was due to the start of the acquisition in the late arterial phase. In 3 of these cases, incorrect determination of the $t_{\mathrm{OA}}$ and $t_{\mathrm{PV}}$ from ROIs placed to include bone, allowed small-scale patient motion to cause severe degradation of the time-enhancement curves. Given the relatively high radiation dose associated with this procedure, it is essential to verify that the shapes of the time-enhancement curves are appropriate before measurement. In the final case, the ROIs were correctly placed, but the $t_{\mathrm{PV}}$ was measured from a broad plateau, in which peak enhancement was early within this plateau.

No discordant results were found in the measured-duration group; this finding may be a reflection of increased experience with the interpretation of $4 \mathrm{D}-\mathrm{CTA} .{ }^{17}$ In addition, the sample size for this group was smaller. This article reports 2 false-positives within the estimated-exposure group. In both cases, the $4 \mathrm{D}$-CTA did not demonstrate early venous enhancement, but suspicion had been raised on the basis of the "prominence" of the veins adjacent to a hematoma. These false-positives could potentially be regarded as interpretation errors, rather than a limitation of the image acquisition.

Methodologic limitations included the definition of adequate venous enhancement by an arbitrary threshold. Enhancement was also measured on postprocessed, subtracted whole-brain maximumintensity-projection images computed by subtracting the mask acquisition from each postcontrast volume. Because maximum enhancement was reported, there is a potential source of bias because the maximum-intensity-projection images can capture the peak values of noise voxels within the projection, causing sensitivity to the through-plane length of the vessel and therefore to the location of ROIs and anatomic configuration.

Note:-NS indicates not stated.
a Ten-second continuous exposure followed by 4 intermittent exposures.
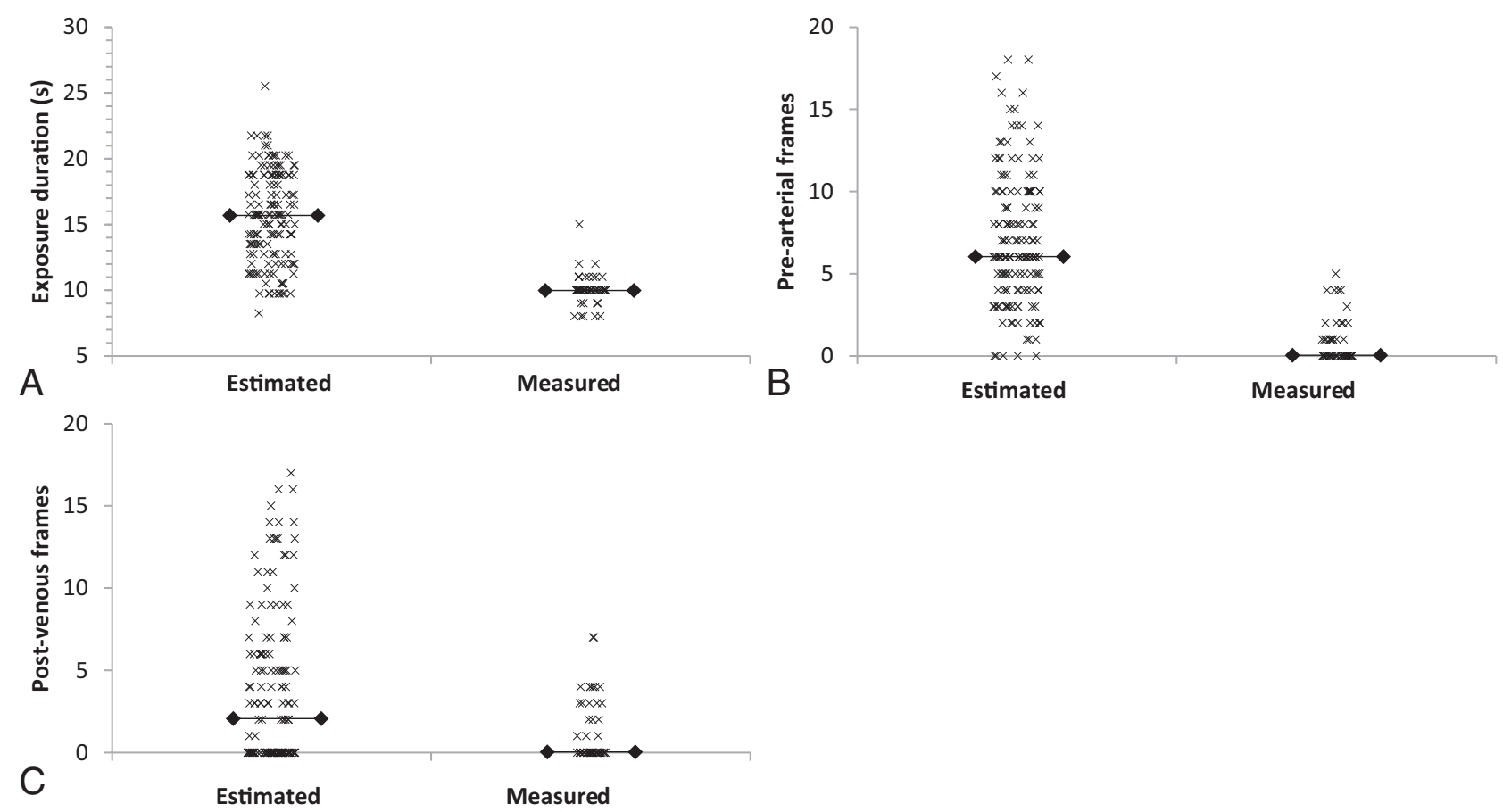

FIG 3. Scatterplots indicating exposure duration $(A)$ and the number of nondiagnostic volumes due to the start of the acquisition before contrast arrival $(B)$ and the end of the acquisition following contrast washout $(C)$. Horizontal lines indicate the median. 


\section{CONCLUSIONS}

We have demonstrated that there is potential for reducing the radiation dose of $4 \mathrm{D}$-CTA by more precisely matching the exposure duration to cerebral circulation time as determined by arterial and venous time-enhancement curves. This finding may be of particular value when combined with higher temporal resolution techniques.

\section{ACKNOWLEDGMENTS}

The authors thank Debasree Purkayastha for advice on statistics and data presentation and for helpful comments about the manuscript.

\section{REFERENCES}

1. Willems PW, Brouwer PA, Barfett JJ, et al. Detection and classification of cranial dural arteriovenous fistulas using 4D-CT angiography: initial experience. AJNR Am J Neuroradiol 2011;32: 49-53 CrossRef Medline

2. Brouwer PA, Bosman T, van Walderveen MA, et al. Dynamic 320section CT angiography in cranial arteriovenous shunting lesions. AJNR Am J Neuroradiol 2010;31:767-70 CrossRef Medline

3. Fujiwara H, Momoshima S, Akiyama T, et al. Whole-brain CT digital subtraction angiography of cerebral dural arteriovenous fistula using 320-detector row CT. Neuroradiology 2013;55:837-43 CrossRef Medline

4. Beijer TR, van Dijk EJ, de Vries J, et al. 4D-CT angiography differentiating arteriovenous fistula subtypes. Clin Neurol Neurosurg 2013; 115:1313-16 CrossRef Medline

5. Willems PW, Taeshineetanakul P, Schenk B, et al. The use of 4D-CTA in the diagnostic work-up of brain arteriovenous malformations. Neuroradiology 2012;54:123-31 CrossRef Medline

6. Frölich AM, Wolff SL, Psychogios MN, et al. Time-resolved assessment of collateral flow using 4D CT angiography in large-vessel occlusion stroke. Eur Radiol 2014;24:390-96 CrossRef Medline

7. Frölich AM, Schrader D, Klotz E, et al. 4D CT angiography more closely defines intracranial thrombus burden than single-phase CT angiography. AJNR Am J Neuroradiol 2013;34:1908-13 CrossRef Medline

8. Ringelstein A, Lechel U, Fahrendorf DM, et al. Radiation exposure in perfusion CT of the brain. J Comput Assist Tomogr 2014;38:25-28 CrossRef Medline

9. Imanishi Y, Fukui A, Niimi H, et al. Radiation-induced temporary hair loss as a radiation damage only occurring in patients who had the combination of MDCT and DSA. Eur Radiol 2005;15:41-46 CrossRef Medline

10. D’Orazio F, Splendiani A, Gallucci M. 320-Row detector dynamic 4D-CTA for the assessment of brain and spinal cord vascular shunting malformations: a technical note. Neuroradiol J 2014;27:710-17 CrossRef Medline

11. Hoogenboom TC, van Beurden RM, van Teylingen B, et al. Optimization of the reconstruction interval in neurovascular 4D-CTA imaging: a technical note. Interv Neuroradiol 2012;18:377-79 Medline

12. Das K, Biswas S, Roughley S, et al. 3D CT cerebral angiography technique using a 320-detector machine with a time density curve and low contrast medium volume: comparison with fixed time delay technique. Clinical Radiol 2014;69:e129-35 CrossRef Medline

13. Biswas $\mathrm{S}$, Chandran A, Roughley $\mathrm{S}$, et al. Cerebral CT venography using a 320-MDCT scanner with a time-density curve technique and low volume of contrast agent: comparison with fixed time-delay technique. AJR Am J Roentgenol 2015;205:1269-75 CrossRef Medline

14. European Commission. European Guidelines for Quality Criteria for Computed Tomography. Luxembourg: European Commission; 2000. Report EUR 16262 EN.

15. Lin $\mathrm{CJ}, \mathrm{Wu} \mathrm{TH}$, Lin $\mathrm{CH}$, et al. Can iterative reconstruction improve imaging quality for lower radiation CT perfusion? Initial experience. AJNR Am J Neuroradiol 2013;34:1516-21 CrossRef Medline

16. Bahner ML, Bengel A, Brix G, et al. Improved vascular opacification in cerebral computed tomography angiography with $80 \mathrm{kVp}$. Invest Radiol 2005;40:229-34 Medline

17. Biswas S, Chandran A, Radon M, et al. Accuracy of four-dimensional CT angiography in detection and characterisation of arteriovenous malformations and dural arteriovenous fistulas. Neuroradiol J2015; 28:376-84 CrossRef Medline 